\title{
Erratum to: Type IV collagen drives alveolar epithelial-endothelial association and the morphogenetic movements of septation
}

\author{
Maria Loscertales ${ }^{1,2^{*}}$, Fotini Nicolaou' ${ }^{1}$, Marion Jeanne ${ }^{3}$, Mauro Longoni ${ }^{1,2}$, Douglas B. Gould ${ }^{3}$, Yunwei Sun ${ }^{1}$, \\ Faouzi I. Maalouf ${ }^{1}$, Nandor Nagy ${ }^{1,2,4}$ and Patricia K. Donahoe ${ }^{1,2,5}$
}

\section{Erratum}

Unfortunately, the original version of this article [1] contained errors. Figure 1 was included incorrectly. The corrected Fig. 1 has been corrected in the original article and Fig. 1 is included correctly below.

Additionally there are minor typographical errors stated below:

Additional file 3: Figure S3 has been revised: The image in the lefthand panel of the 3rd row of Additional file 3: Figure S3 (w/ y-axis labeled "PSPC PDPL") was wrong.

Page 4, second paragraph, third sentence: "week" should be "weak", and the figure panel mentioned last in that sentence should read, "(Fig. 1i-k), not g-i.

The correct sentence is below:

"Col4a1mRNA expression was very weak at epithelial tips and surrounding mesenchyme at E16.5 (Fig. 1h), but later, at E18.5, P6, and P30, it was clearly found in the lung interstitium and at the tips of the developing septa (Fig. 1i-k)."

Page 8, first paragraph, first sentence contains a split infinitive; it should read, "To show abnormalities more clearly in the development of type I pneumonocytes...".

Page 11, third paragraph, the word "defects" is missing, which alters the nuance, making this sentence confusing. The sentence should read: "The absence of branching defects may be because we analyzed heterozygous mutants in which sufficient levels of native protein...".

\section{Additional file}

Additional file 3: Figure S3. (A-D) Histology of R26-Cre ${ }^{E R} ; \mathrm{CO}_{4 a 1^{+/ F l e x 41}}$ and Tie2-Cre; Col4a1 +Flex41 mutants, and (E-I) immunohistochemistry analysis of PDPL-PSPC, HOPX, a-SMA, tropoelastin, and elastin in R26-Cre ${ }^{E R}$; Col4a1 +/Flex41. (A, B) Hematoxylin and eosin shows that both R26-Cre ${ }^{E R}$; Col4a1 ${ }^{+/ \text {Flex41 }}$ and Tie2-Cre; Col4a1 ${ }^{+/ F l e x 41}$ mutants have simplified alveolarization. (C) R26-Cre ${ }^{E R}$; Col4al +/Flex41 septa are thick and with numerous blood capillaries (arrowheads) and cells with lipid content (arrows). (D) Tie2-Cre; Col4a1 +/Flex4l septa are small and short with increases in blood capillaries (arrowheads), but not in cells with lipid content. (E) PSPC and PDPL co-staining shows a disorganized alveolar epithelium. (F) R26-Cre ${ }^{E R}$; Col4a1 +/Flex41 display a decrease of type I pneumocytes as shown by nuclear staining of HOPX. $(G-I)$ Abnormal localization of a-SMA, tropoelastin and elastin in the septa of R26-Cre ${ }^{E R}$; Col4a1 +/Flex41 lungs. Scale bars $=200 \mu \mathrm{m}$ in A and B, $35 \mu \mathrm{m}$ in $\mathrm{C}$ and $\mathrm{D}, 50 \mu \mathrm{m}$ in $\mathrm{E}$, and $100 \mu \mathrm{m}$ in F to I. (TIF $24706 \mathrm{~kb}$ )

\section{Author details}

${ }^{1}$ The Pediatric Surgical Research Laboratories, Massachusetts General Hospital, Boston, MA 02114, USA. ${ }^{2}$ Department of Surgery, Harvard Medical School, Boston, MA 02115, USA. ${ }^{3}$ Departments of Ophthalmology and Anatomy, Institute for Human Genetics, University of California, San Francisco, School of Medicine, San Francisco, CA 94143, USA. ${ }^{4}$ Department of Human Anatomy, Histology and Embryology, Faculty of Medicine,

Semmelweis University, Budapest 1094, Hungary. ${ }^{5}$ Broad Institute of MIT and Harvard, Cambridge, MA 02142, USA.

Published online: 01 September 2016

\section{Reference}

1. Loscertales M, Nicolaou F, Jeanne M, et al. BMC Biol. 2016;14:59. doi:10.1186/s12915-016-0281-2.

\footnotetext{
* Correspondence: MLOSCERTALES@mgh.harvard.edu

${ }^{1}$ The Pediatric Surgical Research Laboratories, Massachusetts General Hospital, Boston, MA 02114, USA

${ }^{2}$ Department of Surgery, Harvard Medical School, Boston, MA 02115, USA

Full list of author information is available at the end of the article
} 


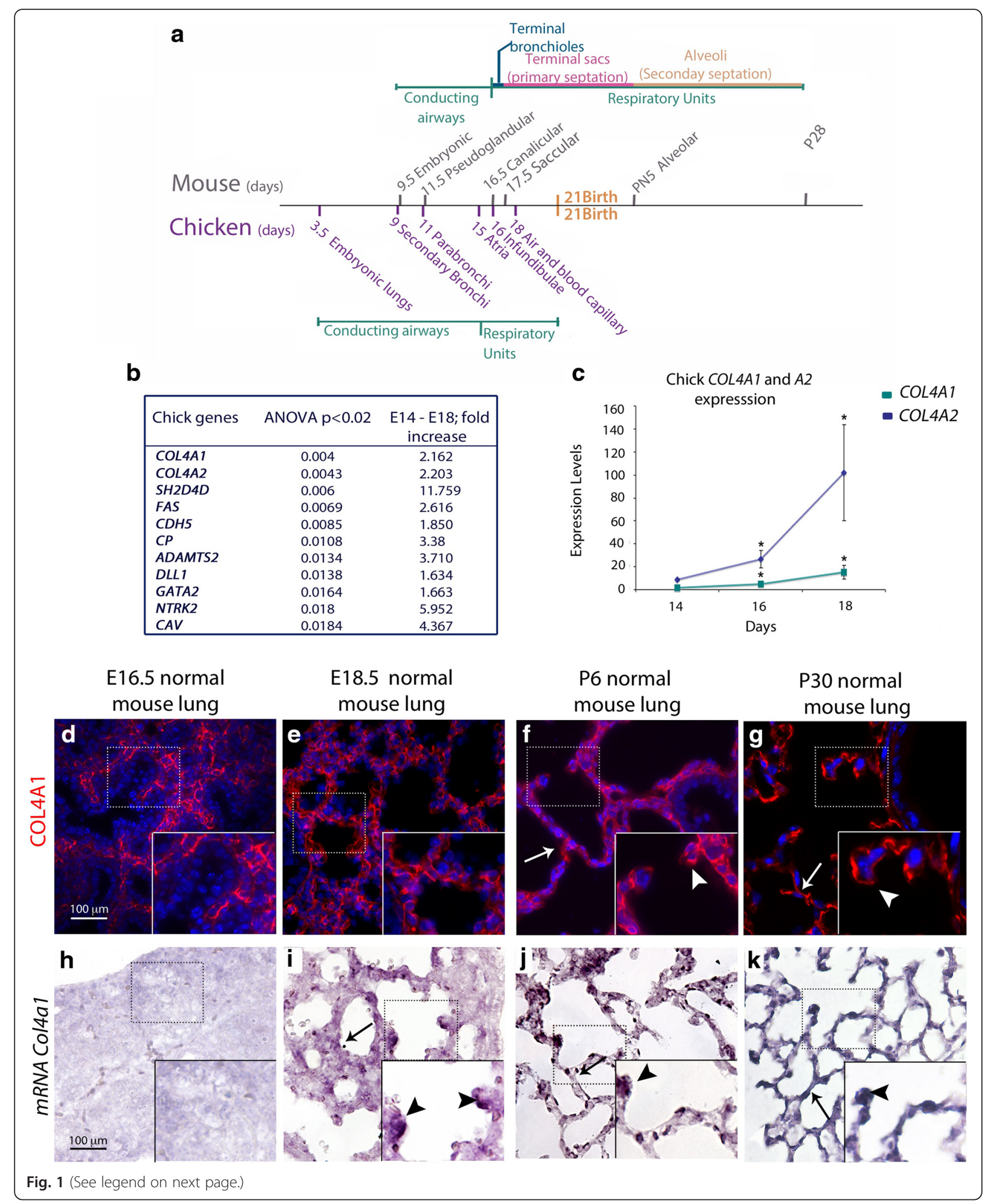


(See figure on previous page.)

Fig. 1 Lung development timeline and type IV collagen expression in the chicken and the mouse. a Mouse and chicken lung development comparative timeline. b Microarray analysis shows that vascular related genes, among which are Co/4a1 and Col4a2, show the highest significance in late chick lung development. c Real-time PCR shows differential expression of Col4al (blue) and Col4a2 (green) between E16 and E18 in chick lungs. Col4a1 and Col4a2 expression increases at E16 and E18, and is statistically significant (Wilcoxon rank-sum test $P<0.05$ ) when compared to E14. Chicken G6PDH was used as a normalizer. $\mathbf{d}-\mathbf{i}$ Type IV collagen protein and mRNA expression in the lung. $\mathbf{d}-\mathbf{g}$ COL4A1 protein is found throughout the lung interstitium and epithelium (arrows) in the prenatal (E18.5) and postnatal (P6, P30) lungs but mainly in the interstitium at E16.5. At P6 and P30 murine COL4A1 protein is found at the tips of secondary septa (arrowhead in FGF). $\mathbf{h}-\mathbf{k}$ Col4a1 mRNA is almost undetectable by in situ hybridization at E16.5 (h) and is later found in a patchy distribution throughout the interstitium (arrows in $\mathbf{i}-\mathbf{k}$ ) with high expression at the tips of primary and secondary septa (arrowheads). Scale bar $=100 \mu \mathrm{m}$ and applies to $\mathbf{d}-\mathbf{i}$ 